\title{
Solving the Maxwell equations by the Chebyshev method: A one-step finite-difference time-domain algorithm
}

\author{
H. De Raedt*, K. Michielsen ${ }^{\dagger}$, J.S. Kole ${ }^{\ddagger}$, M.T. Figge ${ }^{\S}$ \\ Centre for Theoretical Physics and Materials Science Centre \\ University of Groningen, Nijenborgh 4 \\ NL-9747 AG Groningen, The Netherlands
}

(Dated: March 23, 2018)

\begin{abstract}
We present a one-step algorithm that solves the Maxwell equations for systems with spatially varying permittivity and permeability by the Chebyshev method. We demonstrate that this algorithm may be orders of magnitude more efficient than current finite-difference time-domain algorithms.
\end{abstract}

\section{INTRODUCTION}

Most finite-difference time-domain (FDTD) calculations solve the time-dependent Maxwell equations using algorithms based on a proposal by Yee [1, 2, 3. The Yee algorithm is flexible, fast and easy to implement. A limitation of Yee-based FDTD techniques is that their stability is conditional, meaning that their numerical stability depends on the mesh size used for the spatial discretization and on the time step of the time integration [2, 3]. In practice, the amount of computational work required to solve the time-dependent Maxwell equations by present FDTD techniques [2, 3, 4, 5, 6, 7, 8, 9, 10 prohibits applications to a class of important fields such as bioelectromagnetics and VLSI design [2, 11, 12]. The basic reason for this is that the time step in the FDTD calculation has to be relatively small in order to maintain a reasonable degree of accuracy in the time integration.

In this paper we describe a one-step algorithm, based on Chebyshev polynomial expansions 13, 14, 15, 16, 17, 18, to solve the time-dependent Maxwell equations for arbitrarily long times. We demonstrate that the computational efficiency of this one-step algorithm can be orders of magnitude larger than of other FDTD techniques.

\section{ALGORITHM}

We consider EM fields in linear, isotropic, nondispersive and lossless materials. The time evolution of EM fields in these systems is governed by the time-dependent Maxwell equations [19]. Some important physical symmetries of the Maxwell equations can be made explicit by introducing the fields

$$
\mathbf{X}(t) \equiv \sqrt{\mu} \mathbf{H}(t) \quad \text { and } \quad \mathbf{Y}(t) \equiv \sqrt{\varepsilon} \mathbf{E}(t) .
$$

Here, $\mathbf{H}(t)=\left(H_{x}(\mathbf{r}, t), H_{y}(\mathbf{r}, t), H_{z}(\mathbf{r}, t)\right)^{T}$ denotes the magnetic and $\mathbf{E}(t)=\left(E_{x}(\mathbf{r}, t), E_{y}(\mathbf{r}, t), E_{z}(\mathbf{r}, t)\right)^{T}$ the electric field vector, while $\mu=\mu(\mathbf{r})$ and $\varepsilon=\varepsilon(\mathbf{r})$ denote, respectively, the permeability and the permittivity. In the absence of electric charges, Maxwell's curl equations [2] read

$$
\frac{\partial}{\partial t}\left(\begin{array}{c}
\mathbf{X}(t) \\
\mathbf{Y}(t)
\end{array}\right)=\mathcal{H}\left(\begin{array}{c}
\mathbf{X}(t) \\
\mathbf{Y}(t)
\end{array}\right)-\frac{1}{\sqrt{\varepsilon}}\left(\begin{array}{c}
0 \\
\mathbf{J}(t)
\end{array}\right)
$$

where $\mathbf{J}=\left(J_{x}(\mathbf{r}, t), J_{y}(\mathbf{r}, t), J_{z}(\mathbf{r}, t)\right)^{T}$ represents the source of the electric field and $\mathcal{H}$ denotes the operator

$$
\mathcal{H} \equiv\left(\begin{array}{cc}
0 & -\frac{1}{\sqrt{\mu}} \nabla \times \frac{1}{\sqrt{\varepsilon}} \\
\frac{1}{\sqrt{\varepsilon}} \nabla \times \frac{1}{\sqrt{\mu}} & 0
\end{array}\right) .
$$

Writing $\mathbf{Z}(t)=(\mathbf{X}(t), \mathbf{Y}(t))^{T}$ it is easy to show that $\mathcal{H}$ is skew symmetric, i.e. $\mathcal{H}^{T}=-\mathcal{H}$, with respect to the inner product $\left\langle\mathbf{Z} \mid \mathbf{Z}^{\prime}\right\rangle \equiv \int_{V} \mathbf{Z}^{T} \cdot \mathbf{Z}^{\prime} d \mathbf{r}$, where $V$ denotes the system's volume. In addition to Eq.(2), the EM fields also satisfy $\nabla \cdot(\sqrt{\mu} \mathbf{X}(t))=0$ and $\nabla \cdot(\sqrt{\varepsilon} \mathbf{Y}(t))=0$.

\footnotetext{
* E-mail: deraedt@phys.rug.nl

$\dagger$ E-mail: kristel@phys.rug.nl

¥ E-mail: j.s.kole@phys.rug.nl

$\S$ E-mail: m.t.figge@phys.rug.nl

http://www.compphys.rug.nl/
} 
A numerical algorithm that solves the time-dependent Maxwell equations necessarily involves some discretization procedure of the spatial derivatives in Eq. (2). Ideally, this procedure should not change the basic symmetries of the Maxwell equations. We will not discuss the (important) technicalities of the spatial discretization (we refer the reader to Refs. [9, 10]) as this is not essential to the construction of the one-step algorithm.

On a spatial grid Maxwell's curl equations (2) can be written in the compact form [9, 10]

$$
\frac{\partial}{\partial t} \boldsymbol{\Psi}(t)=H \boldsymbol{\Psi}(t)-\boldsymbol{\Phi}(t) .
$$

The vector $\mathbf{\Psi}(t)$ is a representation of $\mathbf{Z}(t)$ on the grid. The matrix $H$ is the discrete analogue of the operator (3), and the vector $\mathbf{\Phi}(t)$ contains all the information on the current source $\mathbf{J}$. The formal solution of Eq. (何) is given by

$$
\boldsymbol{\Psi}(t)=U(t) \mathbf{\Psi}(0)-\int_{0}^{t} U(t-u) \boldsymbol{\Phi}(u) d u,
$$

where $U(t)=e^{t H}$ denotes the time-evolution matrix. The underlying physical symmetries of the time-dependent Maxwell equations are reflected by the fact that the matrix $H$ is real and skew symmetric [9], implying that $U(t)$ is orthogonal [20].

Numerically, the time integration is carried out by using a time-evolution operator $\bar{U}(t)$ that is an approximation to $U(t)=e^{t H}$. We denote the approximate solution by $\overline{\mathbf{\Psi}}(t)$. First we use the Chebyshev polynomial expansion to approximate $U(t)$ and then show how to treat the source term in Eq. (5). We begin by "normalizing" the matrix $H$. The eigenvalues of the skew-symmetric matrix $H$ are pure imaginary numbers. In practice $H$ is sparse so it is easy to compute $\|H\|_{1} \equiv \max _{j} \sum_{i}\left|H_{i, j}\right|$. Then, by construction, the eigenvalues of $B \equiv-i H /\|H\|_{1}$ all lie in the interval $[-1,1]$ [20]. Expanding the initial value $\boldsymbol{\Psi}(0)$ in the (unknown) eigenvectors $\mathbf{b}_{j}$ of $B$, we find from Eq. (5) with $\boldsymbol{\Phi}(t) \equiv 0$ :

$$
\boldsymbol{\Psi}(t)=e^{i z B} \mathbf{\Psi}(0)=\sum_{j} e^{i z b_{j}} \mathbf{b}_{j}\left\langle\mathbf{b}_{j} \mid \Psi(0)\right\rangle
$$

where the $b_{j}$ denote the (unknown) eigenvalues of $B$. Although there is no need to know the eigenvalues and eigenvectors of $B$ explicitly, the current mathematical justification of the Chebyshev approach requires that $B$ is diagonalizable and that its eigenvalues are real. The effect of relaxing these conditions on the applicability of the Chebyshev approach is left for future research. We find the Chebyshev polynomial expansion of $U(t)$ by computing the expansion coefficients of each of the functions $e^{i z b_{j}}$ that appear in Eq. (6). In particular, as $-1 \leq b_{j} \leq 1$, we can use the expansion 21$] e^{i z b_{j}}=J_{0}(z)+2 \sum_{k=1}^{\infty} i^{k} J_{k}(z) T_{k}\left(b_{j}\right)$, where $J_{k}(z)$ is the Bessel function of integer order $k$ to write Eq. (6) as

$$
\boldsymbol{\Psi}(t)=\left[J_{0}(z) I+2 \sum_{k=1}^{\infty} J_{k}(z) \widetilde{T}_{k}(B)\right] \boldsymbol{\Psi}(0) .
$$

Here, $I$ is the identity matrix and $\widetilde{T}_{k}(B)=i^{k} T_{k}(B)$ is a matrix-valued modified Chebyshev polynomial that is defined by the recursion relations

$$
\widetilde{T}_{0}(B) \boldsymbol{\Psi}(0)=\boldsymbol{\Psi}(0), \quad \widetilde{T}_{1}(B) \boldsymbol{\Psi}(0)=i B \boldsymbol{\Psi}(0),
$$

and

$$
\widetilde{T}_{k+1}(B) \Psi(0)=2 i B \widetilde{T}_{k}(B) \Psi(0)+\widetilde{T}_{k-1}(B) \Psi(0),
$$

for $k \geq 1$. In practice we truncate the sum in Eq. (7), i.e. to obtain the approximation $\overline{\mathbf{\Psi}}(t)$ we will sum only the contributions with $k \leq K$. As $\left\|\widetilde{T}_{k}(B)\right\|_{1} \leq 1$ by construction and $\left|J_{k}(z)\right| \leq|z|^{k} / 2^{k} k$ ! for $z$ real [21], the resulting error vanishes exponentially fast for sufficiently large $K$. In Fig. 1] we show a plot of $J_{n}(z=200)$ as a function of $n$ to illustrate this point. From Fig. 1 it is clear that the Chebyshev polynomial expansion will only be useful if $K$ lies to the right of the right-most extremum of $J_{n}(z=200)$. From numerical analysis it is known that for fixed $K$, the Chebyshev polynomial is very nearly the same polynomial as the minimax polynomial [22], i.e. the polynomial of degree $K$ that has the smallest maximum deviation from the true function, and is much more accurate than for instance a Taylor expansion of the same degree $K$. The coefficients $J_{k}(z)$ should be calculated to high precision and the number $K$ is fixed by requiring that $\left|J_{k}(z)\right|<\kappa$ for all $k>K$. Here, $\kappa$ is a control parameter that determines the accuracy of the approximation. For fixed $\kappa, K$ increases linearly with $z=t\|H\|_{1}$ (there is no requirement on $t$ being 
small), a result that is essential for the efficiency of the algorithm. Using the recursion relation of the Bessel functions, all $K$ coefficients can be obtained with $\mathcal{O}(\mathcal{K})$ arithmetic operations 22. Clearly this is a neglible contribution to the total computational cost for solving the Maxwell equations.

Performing one time step amounts to repeatedly using recursion (9) to obtain $\widetilde{T}_{k}(B) \Psi(0)$ for $k=2, \ldots, K$, multiply the elements of this vector by $J_{k}(z)$ and add all contributions. This procedure requires storage for two vectors of the same length as $\boldsymbol{\Psi}(0)$ and some code to multiply such a vector by the sparse matrix $H$. The result of performing one time step yields the solution at time $t$, hence the name one-step algorithm. In contrast to what Eqs. (8) and (9) might suggest, the algorithm does not require the use of complex arithmetic.

We now turn to the treatment of the current source $\mathbf{J}(t)$. The contribution of the source term to the EM field at time $t$ is given by the last term in Eq. (5). One approach might be to use the Chebyshev expansion (76) for $U(t-u)=e^{(t-u) H}$ and to perform the integral in Eq. (5) numerically. However that is not efficient as for each value of $t-u$ we would have to perform a recursion of the kind Eq. (9). Thus, it is better to adopt another strategy. For simplicity we only consider the case of a sinusoidal source

$$
\mathbf{J}(\mathbf{r}, t)=\Theta(T-t) \mathbf{s}(\mathbf{r}) \sin (\Omega t)
$$

where $\mathbf{s}(\mathbf{r})$ specifies the spatial distribution and $\Omega$ the angular frequency of the source. The step function $\Theta(T-t)$ indicates that the source is turned on at $t=0$ and is switched off at $t=T$. Note that Eq. (10) may be used to compose sources with a more complicated time dependence by a Fourier sine transformation.

The formal solution for the contribution of the sinusoidal source (10) reads

$$
\begin{aligned}
\int_{0}^{t} e^{(t-u) H} \boldsymbol{\Phi}(u) d u & =\left(\Omega^{2}+H^{2}\right)^{-1} e^{\left(t-T^{\prime}\right) H} \times\left(\Omega e^{T^{\prime} H}-\Omega \cos \Omega T^{\prime}-H \sin \Omega T^{\prime}\right) \boldsymbol{\Xi} \\
& \equiv f\left(H, t, T^{\prime}, \Omega\right) \boldsymbol{\Xi},
\end{aligned}
$$

where $T^{\prime}=\min (t, T)$ and $\boldsymbol{\Phi}(u) \equiv \Theta(T-t) \sin (\Omega t) \boldsymbol{\Xi}$ with $\boldsymbol{\Xi}$ a vector of the same length as $\boldsymbol{\Psi}(0)$ that represents the time-independent, spatial distribution $\mathbf{s}(\mathbf{r})$. The coefficients of the Chebyshev polynomial expansion of the formal solution (11) are calculated as follows. First we repeat the scaling procedure described above and substitute in Eq. (11) $H=i x\|H\|_{1}, t=z /\|H\|_{1}, T^{\prime}=Z^{\prime} /\|H\|_{1}$, and $\Omega=\omega\|H\|_{1}$. Then, we compute the (Fast) Fourier Transform with respect to $x$ of the function $f\left(x, z, Z^{\prime}, \omega\right)$ (which is non-singular on the interval $-1 \leq x \leq 1$ ). By construction, the Fourier coefficients $S_{k}\left(t\|H\|_{1}\right)$ are the coefficients of the Chebyshev polynomial expansion [21.

Taking into account all contributions of the source term with $k$ smaller than $K^{\prime}$ (determined by a procedure similar to the one for $K$ ), the one-step algorithm to compute the EM fields at time $t$ reads

$$
\begin{aligned}
\overline{\mathbf{\Psi}}(t) & =\left[J_{0}\left(t\|H\|_{1}\right) I+2 \sum_{k=1}^{K} J_{k}\left(t\|H\|_{1}\right) \widetilde{T}_{k}(B)\right] \mathbf{\Psi}(0) \\
& +\left[S_{0}\left(t\|H\|_{1}\right) I+2 \sum_{k=1}^{K^{\prime}} S_{k}\left(t\|H\|_{1}\right) \widetilde{T}_{k}(B)\right] \boldsymbol{\Xi}
\end{aligned}
$$

We emphasize that in our one-step approach the time dependence of the source is taken into account exactly, without actually sampling it.

\section{RESULTS}

The following two examples illustrate the efficiency of the one-step algorithm. First we consider a system in vacuum $\left(\varepsilon=\varepsilon_{0}\right.$ and $\left.\mu=\mu_{0}\right)$ which is infinitely large in the $y$ - and $z$-direction, hence effectively one dimensional. The current source (10) is placed at the center of a system of length 250.1 and oscillates with angular frequency $\Omega=2 \pi$ during the time interval $0 \leq t \leq T=4$ [23]. In Table [ we present results of numerical experiments with two different time-integration algorithms. In general, the error of a solution $\widetilde{\boldsymbol{\Psi}}(t)$ as obtained by the FDTD algorithm of Yee 11, 2] or the unconditionally stable FDTD algorithm $T 4 S 2$ [9, 10] is defined by $\Delta(t) \equiv\|\widetilde{\boldsymbol{\Psi}}(t)-\overline{\mathbf{\Psi}}(t)\| /\|\overline{\mathbf{\Psi}}(t)\|$, where $\overline{\mathbf{\Psi}}(t)$ denotes the vector of the EM fields as obtained by the one-step algorithm. The error on the Yee-algorithm result vanishes as $\tau^{2}$ for sufficiently small $\tau$ [且, 2]. However, as Table I shows, unless $\tau$ is made sufficiently small $(\tau \leq 0.0125$ in this example), the presence of the source term changes the quadratic behavior to almost linear. The rigorous bound on the error between the exact and $T 4 S 2$ results tells us that this error should vanish as $\tau^{4}$ [9, 24]. This knowledge 
can be exploited to test if the one-step algorithm yields the exact numerical answer. Using the triangle inequality we can write

$$
\begin{gathered}
\|\mathbf{\Psi}(t)-\overline{\boldsymbol{\Psi}}(t)\| \leq\|\mathbf{\Psi}(t)-\widetilde{\boldsymbol{\Psi}}(t)\|+\|\widetilde{\boldsymbol{\Psi}}(t)-\overline{\mathbf{\Psi}}(t)\| \\
\leq \tau^{4} t C\left(1+\int_{0}^{t}\|\mathbf{J}(u)\| d u\right)+\Delta(t)\|\overline{\mathbf{\Psi}}(t)\|
\end{gathered}
$$

where $C$ is a positive constant 24]. The numerical data in Table f (third column) show that $\Delta(t) \rightarrow 0$ as $\tau^{4}$ and, therefore, we can be confident that the one-step algorithm yields the correct answer within rounding errors. Furthermore, since the results of the one-step algorithm are exact within almost machine precision, in general the solution also satisfies $\nabla \cdot(\sqrt{\mu} \mathbf{X}(t))=0$ and $\nabla \cdot(\sqrt{\varepsilon} \mathbf{Y}(t))=0$ within the same precision. This high precision also allows us to use the one-step algorithm for genuine time stepping with arbitrarily large time steps, this in spite of the fact that strictly speaking, the one-step algorithm is not unconditionally stable.

From Table If it follows that if one finds an error of more than $2.5 \%$ acceptable, one could use the Yee algorithm, though we recommend to use the one-step algorithm because then the time-integration error is neglegible. The Yee algorithm is no competition for the $T 4 S 2$ algorithm if one requires an error of less than $1 \%$, but the $T 4 S 2$ algorithm is not nearly as efficient as the one-step algorithm with respect to the number of required matrix-vector operations.

A more general quantitative analysis of the efficiency can be made using the fact that for an $n$ th-order algorithm ( $n=2$ for the Yee algorithm and $n=4$ for the $T 4 S 2$ algorithm), the error $\Delta(t)$ vanishes no faster with $\tau$ than $\tau^{n} t$. Each time step takes a number $W(n)$ of matrix-vector operations (of the type $\boldsymbol{\Psi}^{\prime} \leftarrow M \boldsymbol{\Psi}$ ), e.g. for a three-dimensional system we have $W(2)=1$ and $W(4)=10$ for the Yee algorithm and the $T 4 S 2$ algorithm, respectively. In practice the actual number of floating point operations carried out by our algorithms agrees with these estimates. The total number of matrix-vector operations it takes to obtain the solution at a reference time $t_{r}$ with error $\Delta_{r}\left(t_{r}\right)$ is then given by $N_{r}=W(n) t_{r} / \tau_{r}$ and thus $\Delta_{r}\left(t_{r}\right) \propto W(n)^{n} t_{r}^{n+1} / N_{r}^{n}$. The number of operations $N$ that it will take to compute the EM fields at time $t$ with accuracy $\Delta(t)$ is then calculated from

$$
N=N_{r}\left(\frac{\Delta_{r}\left(t_{r}\right)}{\Delta(t)}\right)^{1 / n}\left(\frac{t}{t_{r}}\right)^{(n+1) / n}
$$

We note that one numerical reference experiment per $n$ th-order algorithm is sufficient to determine the parameters $N_{r}, \Delta_{r}\left(t_{r}\right)$, and $t_{r}$. While these parameters may be different for different systems, the scaling of $N$ with $t^{3 / 2}$ and with $t^{5 / 4}$, respectively, for second- and fourth-order algorithms, will not be affected. Most importantly, since the number of matrix-vector operations required by the one-step algorithm scales linearly with $t$, it is clear that for long enough times $t$, the one-step algorithm will be orders of magnitude more efficient than the current FDTD methods. In Fig. 2 we show the required number of operations as a function of time $t$ taking, as an example, simulation data of $3 \mathrm{D}$ systems (discussed below) to fix the parameters $N_{r}, \Delta_{r}\left(t_{r}\right)$, and $t_{r}$. We conclude that for longer times none of the FDTD algorithms can compete with the one-step algorithm in terms of efficiency. For $t=20$, the one-step algorithm is a factor of ten faster than the Yee algorithm. Thereby we have disregarded the fact that the Yee algorithm yields results within an error of $0.1 \%$ while the one-step algorithm gives the numerically exact solution.

As the second example we use the one-step algorithm to compute the frequency spectrum of a three-dimensional photonic woodpile [25. This structure, shown in the inset of Fig. 3, possesses a large infrared bandgap and is under current experimental and theoretical investigation [25, 26. To determine all eigenvalues of the corresponding matrix $H$ we follow the procedure described in Refs. [9, 27, 28]. We use random numbers to initialize the elements of the vector $\boldsymbol{\Psi}(0)$. Then we calculate the inner product $F(t)=\langle\boldsymbol{\Psi}(0) \mid \boldsymbol{\Psi}(t)\rangle$ as a function of $t$ and average $f(t)=F(t) / F(0)$ over several realizations of the initial vector $\boldsymbol{\Psi}(0)$. The full eigenmode distribution, $\mathcal{D}(\omega)$, is obtained by Fourier transformation of $f(t)$. In Fig. 3 we show $\mathcal{D}(\omega)$, as obtained by $T 4 S 2$ and the one-step algorithm, with a time step $\tau=0.075$ (set by the largest eigenvalue of $H$ ), a mesh size $\delta=0.1$, and 8192 time steps. For this choice of parameters, the Yee algorithm would be unstable [2, 3] and would yield meaningless results. The T4S2 calculation shows a peak at $\omega=0$. This reflects the fact that, in a strict sense, the $T 4 S 2$ algorithm does not conserve $\nabla \cdot(\sqrt{\mu} \mathbf{X}(t))$ and $\nabla \cdot(\sqrt{\varepsilon} \mathbf{Y}(t))[9,10]$. However, the peak at $\omega=0$ vanishes as $\tau^{4}$. Repeating the $T 4 S 2$ calculation with $\tau=0.01$ yields a $\mathcal{D}(\omega)$ (not shown) that is on top of the result of the one-step algorithm (see Fig. 3) and is in good agreement with band-structure calculations [25]. For $\tau=0.01$ the one-step algorithm is 3.5 times more efficient than $T 4 S 2$. Note that in this example, the one-step algorithm is used for a purpose for which it is least efficient (time-stepping with relatively small time steps). Nevertheless the gain in efficiency is still substantial. In simulations of the scattering of the EM fields from the same woodpile (results not shown), the one-step algorithm is one to two orders of magnitude more efficient than current FDTD algorithms, in full agreement with the error scaling analysis given above. 


\section{CONCLUSION}

We have described a one-step algorithm, based on the Chebyshev polynomial expansions, to solve the timedependent Maxwell equations with spatially varying permittivity and permeability and current sources. In practice this algorithm is as easy to implement as FDTD algorithms. Our error scaling analysis shows and our numerical experiments confirm that for long times the one-step algorithm can be orders of magnitude more efficient than current FDTD algorithms. This opens possibilities to solve problems in computational electrodynamics that are currently intractable.

\section{Acknowledgments}

H.D.R. and K.M. are grateful to T. Iitaka for drawing our attention to the potential of the Chebyshev method and for illuminating discussions.

[1] K.S. Yee, "Numerical Solution of Initial Boundary Value Problems Involving Maxwell's Equations in Isotropic Media", IEEE Transactions on Antennas and Propagation 14, 302 (1966).

[2] A. Taflove and S.C. Hagness, Computational Electrodynamics - The Finite-Difference Time-Domain Method, (Artech House, Boston, 2000).

[3] K.S. Kunz and R.J. Luebbers, Finite-Difference Time-Domain Method for Electromagnetics, (CRC Press, 1993).

[4] See http://www.fdtd.org

[5] F. Zheng, Z. Chen, and J. Zhang, "Towards the development of a three-dimensional unconditionally stable finite-difference time-domain method" IEEE Trans. Microwave Theory and Techniques 48, 1550 (2000).

[6] T. Namiki, "3D ADI-FDTD Method - Unconditionally Stable Time-Domain Algormithm for Solving Full Vector Maxwell's Equations", IEEE Trans. Microwave Theory and Techniques 48, 1743 (2001).

[7] F. Zheng and Z. Chen "Numerical dispersion analysis of the unconditionally stable 3D ADI-FDTD method" IEEE Trans. Microwave Theory and Techniques 49, 1006 (2001).

[8] W. Harshawardhan, Q. Su, and R. Grobe, "Numerical solution of the time-dependent Maxwell's equations for random dielectric media", Phys. Rev. E 62, 8705 (2000).

[9] J.S. Kole, M.T. Figge and H. De Raedt, "Unconditionally Stable Algorithms to Solve the Time-Dependent Maxwell Equations", Phys. Rev. E 64, 066705 (2001).

[10] J.S. Kole, M.T. Figge and H. De Raedt, Phys. Rev. E (in press). "Higher-Order Unconditionally Stable Algorithms to Solve the Time-Dependent Maxwell Equations", Phys. Rev. E 65, 066705-1 (2002).

[11] O.P. Gandi, Advances in Computational Electrodynamics - The Finite-Difference Time-Domain Method, A. Taflove, Ed., (Artech House, Boston, 1998).

[12] B. Houshmand, T. Itoh, and M. Piket-May, Advances in Computational Electrodynamics - The Finite-Difference TimeDomain Method, A. Taflove, Ed., (Artech House, Boston, 1998).

[13] H. Tal-Ezer, "Spectral Methods in Time for Hyperbolic Equations", SIAM J. Numer. Anal. 23, 11 (1986)

[14] H. Tal-Ezer and R. Kosloff, "An accurate and efficient scheme for propagating the time dependent Schödinger equation", J. Chem. Phys. 81, 3967 (1984).

[15] C. Leforestier, R.H. Bisseling, C. Cerjan, M.D. Feit, R. Friesner, A. Guldberg, A. Hammerich, G. Jolicard, W. Karrlein, H.-D. Meyer, N. Lipkin, O. Roncero, and R. Kosloff, "A Comparison of Different Propagation Schemes for the Time Dependent Schrödinger Equation", J. Comp. Phys. 94, 59 (1991).

[16] T. Iitaka, S. Nomura, H. Hirayama, X. Zhao, Y. Aoyagi, and T. Sugano, "Calculating the linear response functions of noninteracting electrons with a time-dependent Schödinger equation", Phys. Rev. E 56, 1222 (1997).

[17] R.N. Silver and H. Röder, "Calculation of densities of states and spectral functions by Chebyshev recursion and maximum entropy", Phys. Rev. E 56, 4822 (1997).

[18] Y.L. Loh, S.N. Taraskin, and S.R. Elliot, "Fast Time-Evolution Method for Dynamical Systems", Phys. Rev. Lett. 84, 2290 (2000); ibid. Phys.Rev.Lett. 84, 5028 (2000).

[19] M. Born and E. Wolf, Principles of Optics, (Pergamon, Oxford, 1964).

[20] J.H. Wilkinson, The Algebraic Eigenvalue Problem, (Clarendon Press, Oxford, 1965).

[21] M. Abramowitz and I. Stegun, Handbook of Mathematical Functions, (Dover, New York, 1964).

[22] W.H. Press, B.P. Flannery, S.A. Teukolsky, and W.T. Vetterling, Numerical Recipes, (Cambridge, New York, 1986).

[23] We measure distances in units of $\lambda$. Time and frequency are expressed in units of $\lambda / c$ and $c / \lambda$, respectively.

[24] H. De Raedt, "Product Formula Algorithms for Solving the Time Dependent Schrödinger Equation", Comp. Phys. Rep. 7, 1 (1987).

[25] S.Y. Lin, J.G. Fleming, D.L. Hetherington, B.K. Smith, R. Biswas, K.M. Ho, M.M. Sigalas, W. Zubrzycki, S.R. Kurtz, and J. Bur, "A three-dimensional photonic crystal operating at infrared wavelengths", Nature 394, 251 (1998). 
[26] J.G. Fleming, S.Y. Lin, I. El-Kady, R. Biswas, and K.M. Ho, "All-metallic three-dimensional photonic crystals with a large Infrared bandgap", Nature 417, 52 (2002).

[27] R. Alben, M. Blume, H. Krakauer, and L. Schwartz, "Exact results for a three-dimensional alloy with site diagonal disorder: comparison with the coherent potential approximation", Phys. Rev. B 12, 4090 (1975).

[28] A. Hams and H. De Raedt, "Fast algorithm for finding the eigenvalue distribution of very large matrices", Phys. Rev. E 62, 4365 (2000). 
TABLE I: The error $\Delta(t)$ after simulation time $t=100$ as a function of the time step $\tau$ for two FDTD algorithms. The number of matrix-vector operations required to compute the solution, is $K^{\prime}=2080, t / \tau$, and $6 t / \tau$ for the one-step, Yee, and $T 4 S 2$ algorithm, respectively.

\begin{tabular}{ccc}
\hline \hline$\tau$ & Yee & $T 4 S 2$ \\
\hline $0.10000 \times 10^{+0}$ & $0.75 \times 10^{-1}$ & $0.51 \times 10^{-1}$ \\
$0.50000 \times 10^{-1}$ & $0.25 \times 10^{-1}$ & $0.33 \times 10^{-2}$ \\
$0.25000 \times 10^{-1}$ & $0.12 \times 10^{-1}$ & $0.21 \times 10^{-3}$ \\
$0.12500 \times 10^{-1}$ & $0.66 \times 10^{-2}$ & $0.13 \times 10^{-4}$ \\
$0.62500 \times 10^{-2}$ & $0.24 \times 10^{-2}$ & $0.91 \times 10^{-6}$ \\
$0.31250 \times 10^{-2}$ & $0.63 \times 10^{-3}$ & $0.30 \times 10^{-6}$ \\
$0.15625 \times 10^{-2}$ & $0.16 \times 10^{-3}$ & $0.15 \times 10^{-7}$ \\
$0.78125 \times 10^{-3}$ & $0.39 \times 10^{-4}$ & $0.60 \times 10^{-8}$ \\
\hline \hline
\end{tabular}

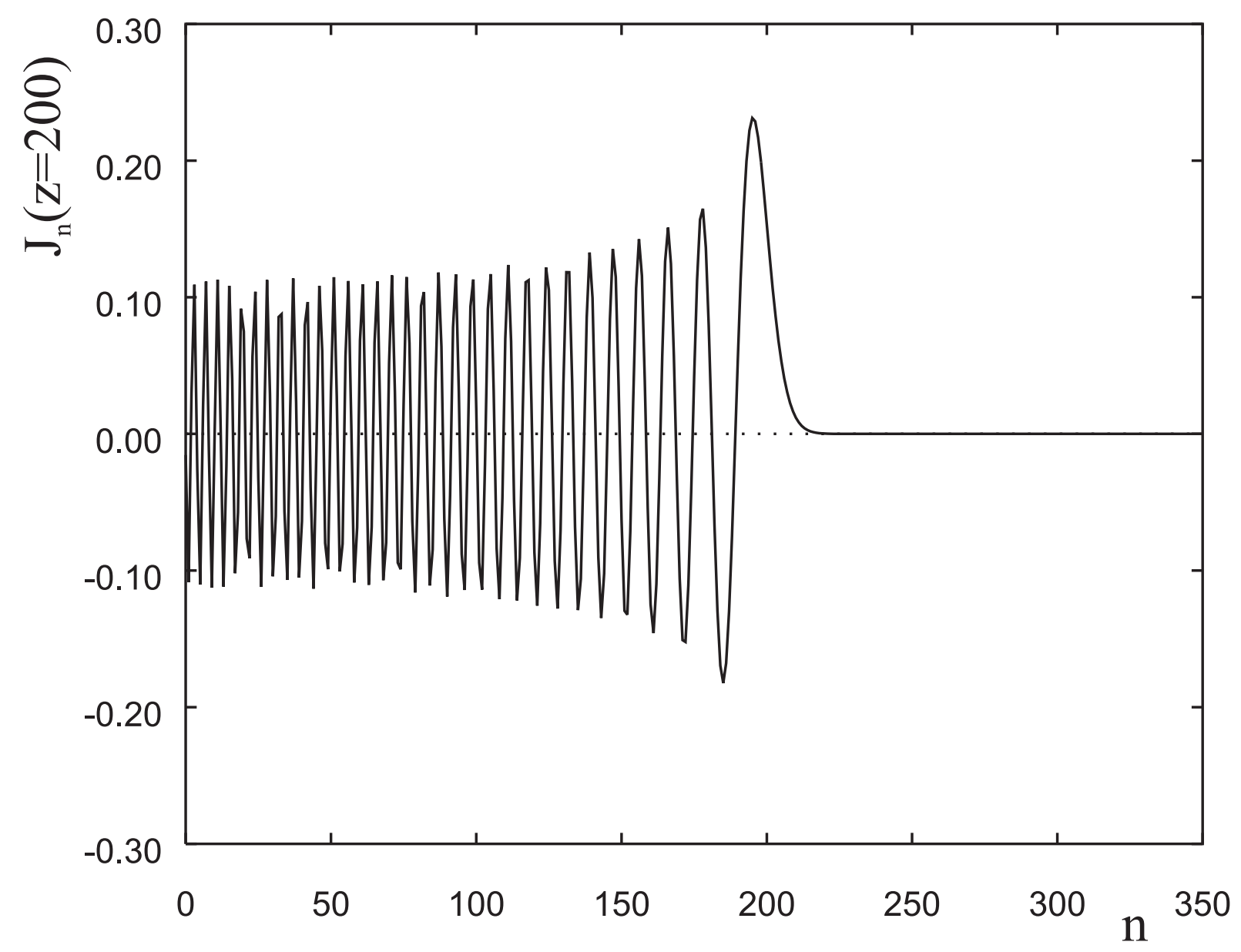

FIG. 1: Dependence of the Bessel function $J_{n}(z=200)$ on the order $n$. 


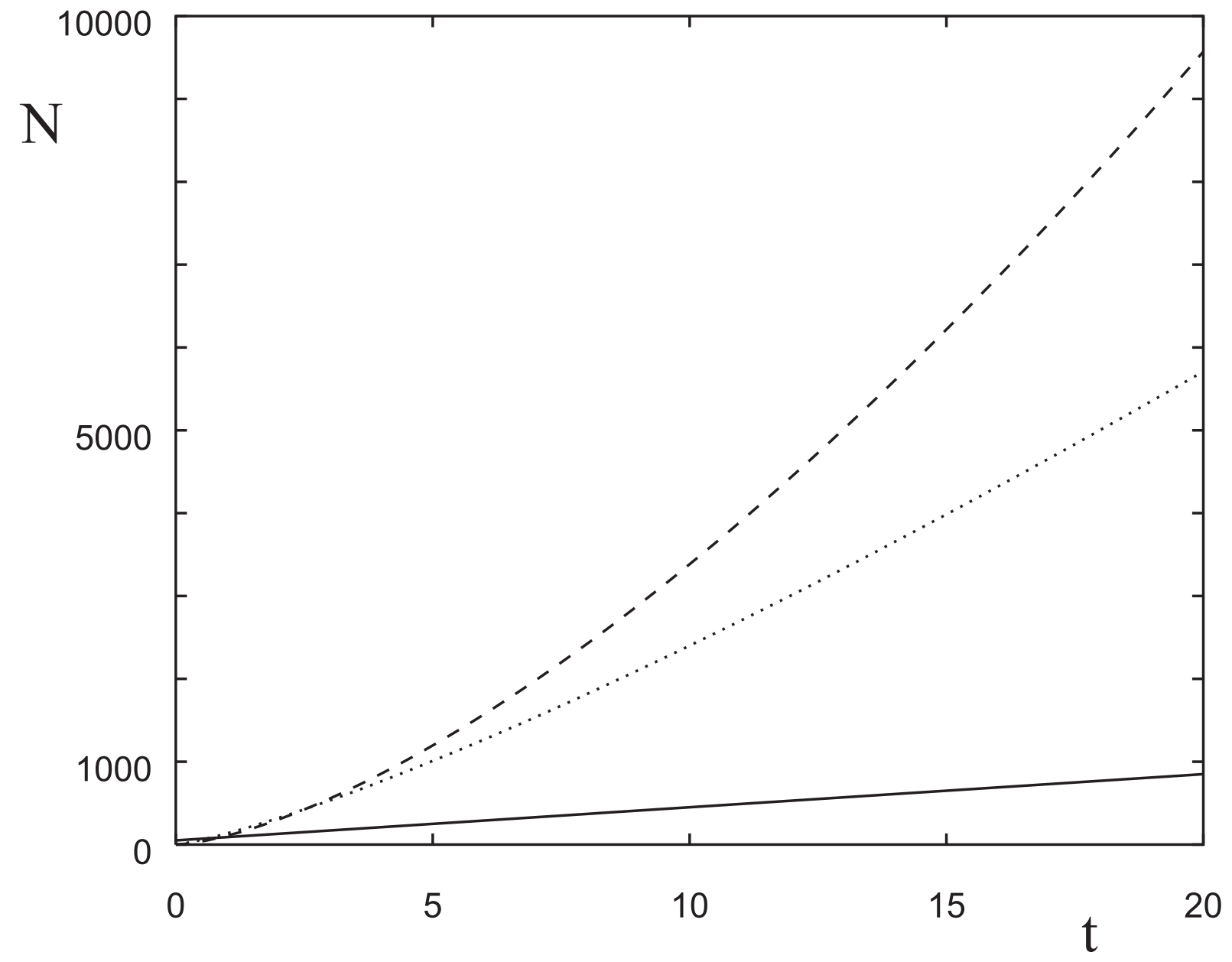

FIG. 2: The number of $\Psi^{\prime} \leftarrow M \Psi$ operations $N$ needed to compute the solution of the 3D Maxwell equation at time $t$ for systems like those shown in Fig.3. Solid line: One-step algorithm; dashed line: Yee algorithm [1, 2, 3] yielding a solution within $0.1 \%$ error; dotted line: T4S2 algorithm [9, 10] yielding a solution within $0.1 \%$ error. 


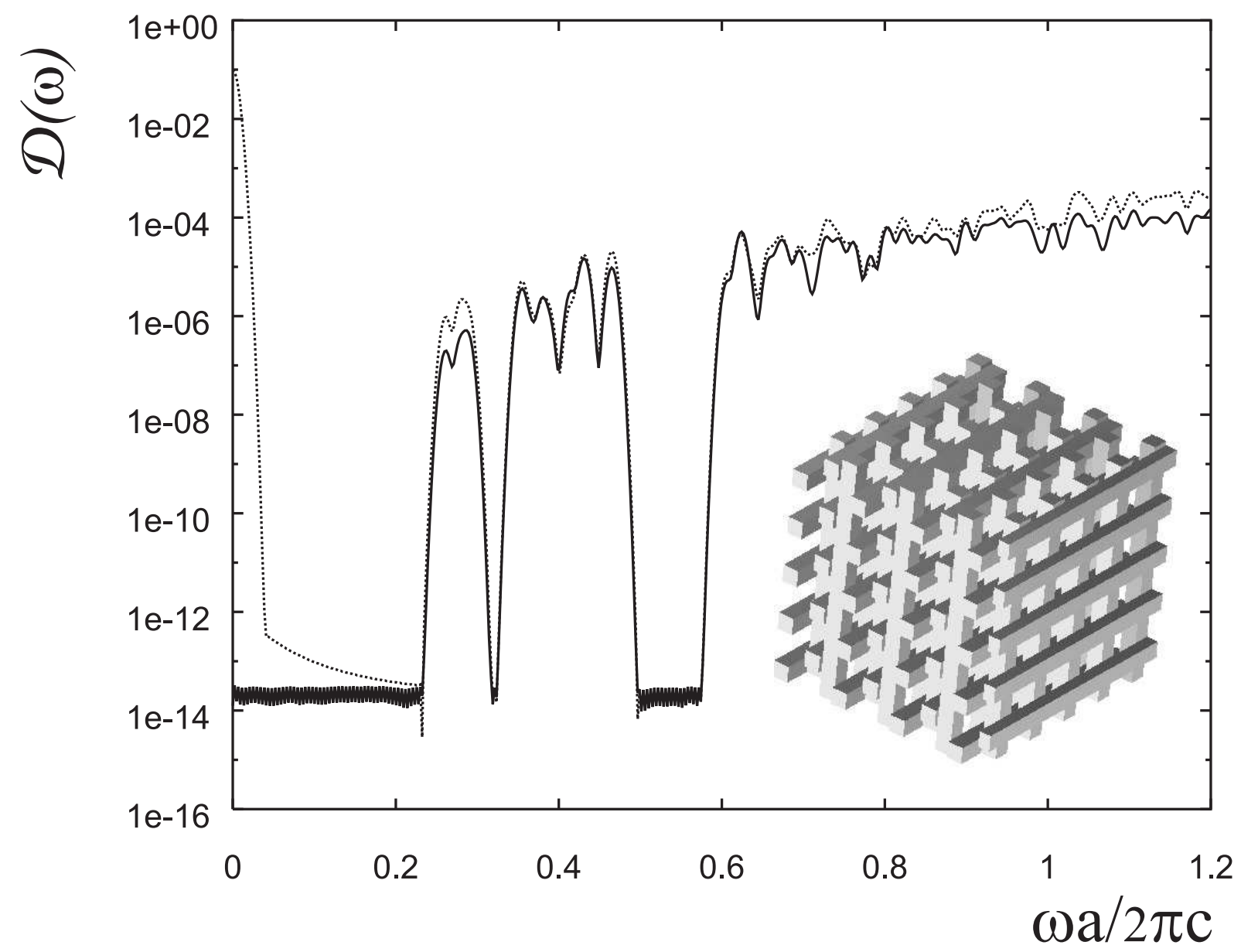

FIG. 3: Frequency spectrum of a three-dimensional photonic woodpile (inset) 25 as obtained by $T 4 S 2$ (dashed line) and the one-step algorithm (solid line). The width, height and period of the rods are $0.55,0.7$, and 2, respectively. The dielectric constant of the rods is 12.96 and the simulation box measures $6 \times 6 \times 5.6$ [23], subject to periodic boundary conditions. 